\title{
The clinical value of autofluorescence bronchoscopy for the diagnosis of lung
}

\section{cancer}

\author{
B. Lam*, M.P. Wong ${ }^{\#}$, S.L. Fung ${ }^{\top}$, D.C.L. Lam*, P.C. Wong ${ }^{\top}$, T.Y.W. Mok ${ }^{+}$, F.M. Lam ${ }^{\top}$, \\ M.S.M. Ip*, C.G.C. Ooi ${ }^{\S}$ and W.K. Lam*
}

ABSTRACT: The aim of this study was to evaluate the role of autofluorescence bronchoscopy (AFB) in the routine work-up of lung cancer.

Consecutive patients with atypical or suspicious cells in sputum or bronchial aspirate, no localising abnormality on chest radiography and nondiagnostic white-light bronchoscopic (WLB) results were recruited. WLB and AFB were performed sequentially during the same session. All abnormal areas detected by WLB, AFB or both were sampled and the biopsy specimens sent for histological examination.

Sixty-two patients were recruited within the 32-month study period. Seventeen had no endobronchial lesion detected. Among the $\mathbf{4 5}$ patients with endobronchial lesions, 37 had lesions with a histopathological grade of mild dysplasia or less; of the eight patients who had a lesion with a histological grade of moderate dysplasia or worse, five were found to have lung cancer, two invasive lung cancer and three an intra-epithelial neoplasm (severe dysplasia). Lesions showing moderate dysplasia or worse were more commonly found in patients with suspicious cells than in those with atypical cells on sputum examination. AFB was more sensitive than WLB (91 versus $58 \%$ ) at detecting these lesions, but less specific (26 versus $50 \%$ ).

A combination of white-light and autofluorescence bronchoscopy can increase the diagnostic yield of this invasive procedure in patients exhibiting abnormal sputum cytology.

KEYWORDS: Autofluorescence bronchoscopy, clinical tool, lung cancer, pre-invasive lesion

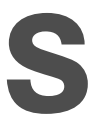
putum cytology is commonly employed as one of the investigations undertaken in patients suspected of having lung cancer, and it is not uncommon for pulmonary physicians to encounter patients with sputum atypia such that further investigation has to be contemplated. Lung cancer arising from the central airways can be detected by means of conventional bronchoscopy. However, patients with carcinoma in situ (CIS), micro-invasive cancers and pre-invasive lesions present a diagnostic challenge, even for experienced bronchoscopists [1]. Since the prognosis of lung cancer patients correlates with the stage of the disease, the importance of early detection followed by early treatment might improve the prognosis in lung cancer. Autofluorescence bronchoscopy (AFB) was developed to enhance the detection of preinvasive lesions, based on the observation that abnormal tissue showed reduced fluorescence compared with normal tissue upon excitation with blue light (wavelength 380-460 nm) [2]. The value of AFB in detecting early lung cancer/pre-invasive lesions has been evaluated in different clinical and research settings [3-5]. However, its role in the routine work-up of lung cancer has not been extensively evaluated. Therefore, the value of AFB was investigated in patients with atypical or suspicious cells in sputum or bronchial aspirate but nondiagnostic white-light bronchoscopic (WLB) results.

\section{MATERIALS AND METHODS}

\section{Study population and eligibility}

Consecutive patients referred to Queen Mary Hospital, the main teaching hospital of the University of Hong Kong (Pokfulam, Hong Kong Special Administrative Region, China), for AFB during the period February 2002 to October 2004 were prospectively recruited. Inclusion criteria included report of: 1) atypical cells or cells suspected of malignancy in sputum or bronchial aspirate; 2) absence of a suspicious lung shadow or infiltrate on plain chest radiographs; and 3) nondiagnostic WLB results. Atypical cells are defined as epithelial cells that

\section{AFFILIATIONS}

Depts of *Medicine

\#Pathology, and

${ }^{\S}$ Diagnostic Radiology, University of Hong Kong,

"Tuberculosis and Chest Unit, Grantham Hospital, and

${ }^{+}$Respiratory Medical Dept, Kowloon Hospital, Hong Kong SAR, China.

CORRESPONDENCE

B. Lam

University Dept of Medicine University of Hong Kong Queen Mary Hospital Pokfulam

Hong Kong SAR

China

Fax: 85228725828

E-mail: lambing@hkucc.hku.hk

Received:

November 092005

Accepted after revision:

June 302006

SUPPORT STATEMENT

The Pentax SAFE-1000

autofluorescence system was

provided by Pentax Asahi Optical Co (Tokyo, Japan) 
show abnormal nuclear features but which are not suspected of being malignant cells. The nuclear changes include mild nuclear enlargement and raised nuclear to cytoplasmic ratio, coarse chromatin with hyperchromasia, and inconspicuous or absent nucleoli. Suspicious cells are defined as epithelial cells that show severe nuclear abnormalities but for which a diagnosis of malignancy cannot be ascertained owing to the limited number of such abnormal cells or coexisting degenerative features [6].

The present study was approved by the Institutional Review Board of the University of Hong Kong.

\section{White-light and autofluorescence bronchoscopy}

Bronchoscopy was performed under local anaesthesia in the endoscopy suite of Queen Mary Hospital by the two investigators (B. Lam and S.L. Fung) who were experienced in AFB. Conventional WLB was performed first, followed by AFB (SAFE-1000: System of Autofluorescence Endoscopy; Pentax, Asahi Optical, Tokyo, Japan). All abnormal lesions detected by WLB, AFB or both were videotaped, and biopsy specimens were taken from these lesions. The location of each biopsy specimen in the bronchial tree was precisely recorded using the international bronchial location classification [7]. Biopsy specimens were fixed in formaldehyde and embedded in paraffin. One expert pathologist (M.P. Wong), without knowledge of the bronchoscopic findings, evaluated the specimens routinely stained with haematoxylin and eosin. The pathological diagnoses were coded according to the World Health Organization lung cancer classification [8].

Patients underwent work-up for resectability if the histopathological results showed invasive cancer. Endobronchial therapy (cryotherapy; ERBE Elektromedizin, Tübingen, Germany) was applied if the histopathological result showed a pre-invasive lesion (severe dysplasia or CIS). For subjects who underwent cryotherapy, re-assessment WLB and AFB were performed at regular intervals. Biopsy specimens were taken from all new and previously treated lesions on re-assessment bronchoscopy. Subjects with moderate dysplasia were followed up in the same way as those who had received cryotherapy. The exact localisation of the previous biopsy specimen was deduced from the initial record during re-assessment bronchoscopy. For those patients for whom no diagnosis was reached after AFB, referring doctors were contacted and clinical progress updated.

\section{TABLE 1 Characteristics of subjects}

$\begin{array}{lc}\text { Age yrs } & 59.9 \pm 12.5(19-83) \\ \text { Male/female } & 44 / 18 \\ \text { Ever-smoker/nonsmoker } & 39 / 23 \\ \text { Sputum finding } & \\ \quad \text { Atypical cell } & 43 \\ \quad \text { Suspected malignancy } & 12 \\ \text { Bronchial aspirate cytology } & \\ \quad \text { Atypical cell } & 5 \\ \quad \text { Suspected malignancy } & 2\end{array}$

Data are presented as mean \pm SD (range) or as absolute values

Informed written consent was obtained from all patients prior to WLB and AFB.

\section{Statistical analysis}

Data are presented as mean $\pm \mathrm{SD}$. The sensitivity, specificity, positive predictive value and negative predictive value of WLB and AFB in detecting moderate dysplasia or worse in the whole group and in the different patient groups (based on degree of sputum or bronchial aspirate abnormality) were calculated separately.

\section{RESULTS}

Patient characteristics

Sixty-two patients fulfilled the inclusion criteria, and their characteristics are shown in table 1 . Twenty patients had underlying lung diseases, including a history of pulmonary tuberculosis, silicosis, bronchiectasis, chronic obstructive lung disease, asthma and resected lung cancer. The indications leading to sputum cytological examination included haemoptysis, chronic cough, imaging finding of lung nodule(s), radiological worsening after several months of anti-tuberculosis treatment, etc. Two subjects had a history of lung cancer; one (left pneumonectomy 17 yrs previously) presented with fever and haemoptysis, and the other (right upper lobectomy 6 yrs previously) underwent sputum sampling during routine follow-up. All subjects underwent normal WLB, except one who had an endobronchial tumour at the lingular bronchus and swollen mucosa found at the left lower lobe bronchus. The lingular tumour was confirmed to be a squamous cell carcinoma, and biopsy of the swollen mucosa over the left

\begin{tabular}{|c|c|c|c|c|c|c|c|c|c|}
\hline & \multirow[t]{2}{*}{ Normal } & \multirow[t]{2}{*}{ Metaplasia } & \multicolumn{3}{|c|}{ Dysplasia } & \multirow[t]{2}{*}{ CIS } & \multirow[t]{2}{*}{ Invasive cancer } & \multirow[t]{2}{*}{ Other } & \multirow[t]{2}{*}{ Total } \\
\hline & & & Mild & Moderate & Severe & & & & \\
\hline AFB & 25 & 6 & 5 & 2 & 3 & & & & 41 \\
\hline Total & 49 & 12 & 10 & 5 & 4 & 2 & 1 & $1^{\#}$ & 84 \\
\hline
\end{tabular}

CIS: carcinoma in situ. ${ }^{\#}$ : endobronchial tuberculosis in this patient. 


\begin{tabular}{|c|c|c|c|c|c|c|c|c|}
\hline \multirow[t]{3}{*}{ TABLE 3} & \multicolumn{8}{|c|}{$\begin{array}{l}\text { Bronchoscopic findings and number of lesions with moderate dysplasia or worse based on degree of cytological } \\
\text { abnormality at baseline }\end{array}$} \\
\hline & & \multirow[t]{2}{*}{ Subjects $n$} & \multicolumn{3}{|c|}{ Lesions detected } & \multicolumn{3}{|c|}{ Moderate dysplasia or worse } \\
\hline & & & WLB & AFB & WLB+AFB & WLB & AFB & WLB+AFB \\
\hline \multicolumn{2}{|c|}{ Atypical sputum cells } & 43 & 14 & 25 & 17 & 1 & 3 & 3 \\
\hline \multicolumn{2}{|c|}{ Suspicious BA cells } & 2 & 0 & 3 & 2 & 0 & 0 & 0 \\
\hline
\end{tabular}

lower lobe bronchus was nondiagnostic. The patient was referred for AFB before lung resection (lobectomy) to rule out synchronous lung cancer.

WLB had been performed at least once in all 62 subjects recruited, and nine had undergone more than one bronchoscopic examination. The median interval from previous bronchoscopic examination to AFB was 2 months.

\section{Bronchoscopic findings}

Seventeen patients showed no detectable lesion on both WLB and AFB. A total of 91 endobronchial lesions were detected in 45 patients. Four specimens were found to be inadequate for diagnosis and two specimens were not reviewed for

\begin{tabular}{|c|c|c|}
\hline \multirow[t]{2}{*}{ TABLE 4} & \multicolumn{2}{|c|}{$\begin{array}{l}\text { Sensitivity, specificity, and positive (PPV) and } \\
\text { negative predictive value (NPV) of white-light } \\
\text { bronchoscopy (WLB) and autofluorescence } \\
\text { bronchoscopy (AFB) in diagnosing } \\
\text { histopathological grade moderate dysplasia or } \\
\text { worse in different patient groups and in all } \\
\text { patients }\end{array}$} \\
\hline & WLB & AFB \\
\hline \multicolumn{3}{|c|}{ Sputum atypia group\# } \\
\hline Sensitivity & $57.1(20-88)$ & $85.7(42-99)$ \\
\hline Specificity & $44.9(31-60)$ & $26.5(15-41)$ \\
\hline PPV & $12.9(4-31)$ & $14.3(6-29)$ \\
\hline NPV & 88.0 (68-99) & $93.0(64-100)$ \\
\hline \multicolumn{3}{|c|}{$\begin{array}{l}\text { Suspicious sputum cells } \\
\text { group }\end{array}$} \\
\hline Sensitivity & $60.0(17-93)$ & $100(46-100)$ \\
\hline Specificity & $58.8(32-81)$ & $35.3(15-61)$ \\
\hline PPV & $30.0(8-65)$ & $31.3(12-59)$ \\
\hline NPV & $83.3(57-97)$ & $100(52-100)$ \\
\hline \multicolumn{3}{|c|}{ Whole group ${ }^{+}$} \\
\hline Sensitivity & $58.3(29-84)$ & $91.7(60-100)$ \\
\hline Specificity & $50.0(38-62)$ & $26.4(17-38)$ \\
\hline PPV & $16.3(7-31)$ & $17.2(9-29)$ \\
\hline NPV & 87.8 (73-95) & $95.0(73-100)$ \\
\hline
\end{tabular}

Data are presented as percentage (95\% confidence interval). ${ }^{*}: 56$ lesions in 33 patients; ${ }^{\bullet}: 22$ lesions in nine patients; ${ }^{+}: 84$ lesions in 45 patients. administrative reasons. The known lingular tumour was excluded from the analysis. Of the remaining 84 lesions, 41 were detected by AFB alone, 20 by WLB alone and 23 by both methods (table 2).

Patients referred with sputum atypia

Of the 43 patients, 10 showed normal bronchoscopic results. A total of 56 lesions were detected in 33 patients (table 3 ).

Patients referred with suspicious cells in sputum

Of the 12 patients, three showed normal bronchoscopic results. A total of 22 lesions were detected in nine patients (table 3 ).

Patients referred with atypical or suspicious cells in bronchial aspirate

Of the seven patients, four showed no endobronchial lesions. A total of six lesions were detected in three patients (table 3). None of the lesions were graded histologically as showing moderate dysplasia or worse.

The patient with a lingular tumour was found to exhibit synchronous severe dysplasia at the lateral subsegment of the superior segment of the right middle lobe (detected by AFB) and CIS at the lateral subsegment of the superior segment of the lingular lobe (detected by both WLB and AFB). Lesions were also detected by AFB at the apical lower segment of the left lower lobe and the lateral subsegment of the lateral basal segment of the left lower lobe, which showed no significant pathology and mild dysplasia, respectively.

The sensitivity, specificity, and positive and negative predictive value of AFB and WLB in detecting moderate dysplasia or worse in different patient groups and in all patients are shown in table 4 .

By using the number of patients as denominator, the diagnostic yield of AFB plus WLB for histopathological grade moderate dysplasia or worse was $12.9 \%$ (eight out of 62 ), and, for severe dysplasia or worse, was $8 \%$ (five out of 62 ).

\section{Computed tomographic findings}

Forty-three patients had undergone computed tomography (CT) of the thorax before AFB and 10 following it. None was found to show radiological evidence of lung cancer. Ten underwent repeat CT during the follow-up period, and one was found to have a slowly growing lung nodule. 


\section{Patient outcome}

After excluding the five patients with pre-invasive lung cancer on AFB plus WLB, the most up-to-date clinical condition of the remaining 57 patients was traced. After a mean follow-up period of $22.2 \pm 10.4$ months, one patient (normal baseline sputum cytological results, normal WLB and AFB results, but atypical cell found in bronchial aspirate) was diagnosed as having stage IA lung cancer by percutaneous needle aspiration of a slowly growing right upper lobe nodule and one patient (sputum contained suspicious cell) was diagnosed as having carcinoma of the vocal cord (vocal cord nodule seen during bronchoscopic examination).

\section{DISCUSSION}

The present study shows that, in this group of subjects who presented with abnormal sputum or bronchial aspirate cytological results and nondiagnostic WLB results, lung cancer/pre-invasive lesions could be detected in $8 \%$ by means of AFB.

Lung cancer is the leading cause of cancer death throughout the world $[9,10]$. In order to improve prognosis, every effort should be made to diagnose lung cancer at its earliest stage. Since high-grade pre-invasive lesions have a high chance of progressing to invasive cancer [11], they should be treated, if possible, and preferably using endobronchial treatment [12]. Currently, the only noninvasive means of diagnosing preinvasive lesions is by sputum examination, and the presence of abnormal cells in sputum or bronchial aspirate is a clear indication for a thorough investigation. In this study, patients were not selected based on their risks of developing lung cancer, i.e. smoking history etc., but according to clinical need.

The two cases of CIS, one case of micro-invasive cancer and one case of severe dysplasia which were not detected by WLB performed in other hospitals, were detected by WLB during the AFB/WLB session in the present study. This could be attributed to disease progression during the time lapse between the two bronchoscopic examinations. It is also possible that bronchoscopists with experience in AFB might have been trained to detect more subtle mucosal changes using WLB.

The detection of synchronous lesions (severe dysplasia and CIS) in the subject pending curative resection led to a change in the management plan (radiotherapy instead of surgery). It is intuitive to suggest that AFB should be considered in the preoperative assessment of patients with operable lung cancer. Indeed, AFB has been used to detect subtle synchronous malignancy and to delineate the extent of lung cancer prior to resection [13-15], although the effect of using AFB on the prognosis of operable lung cancer remains an issue for future evaluation.

There are several limitations to the present study. The original sputum or bronchial aspirate specimens collected before referral were not reviewed due to administrative restraints. It was, therefore, impossible to confirm reported sputum abnormality. However, two cases of lung cancer, one of vocal cord cancer and one of severe dysplasia from among the 12 subjects who had suspicious cells in their sputum, compared with two cases of severe dysplasia from among the 43 subjects who had atypical cells in their sputum would suggest that the baseline reports of sputum cytological abnormality were reasonably accurate. The second limitation is that an adequate explanation could not be provided for all of the detected sputum or bronchial aspirate atypia. However, sputum atypia has been reported in a variety of conditions other than lung cancer, including bronchiectasis, airway inflammation and pulmonary infection [16]. Since a third of the present patients had an underlying lung problem, the present authors believe that the presence of these conditions might have contributed to the detection of the original cytological abnormalities. Another limitation is that random biopsy specimens were not taken from sites which looked normal using both WLB and AFB. Therefore, it is not possible to calculate the sensitivity and specificity of AFB combined with WLB.

Although the role of autofluorescence bronchoscopy in lung cancer screening remains to be determined, it has been shown that the combination of autofluorescence/white-light bronchoscopy can increase the diagnostic yield of this invasive procedure in patients with abnormal sputum cytology. The diagnostic yield seems to be higher in subjects with suspicious cells than in those with atypical cells in sputum. Although some extra time is required for the additional use of autofluorescence bronchoscopy during bronchoscopic examination, it is worthwhile, considering the fact that $8 \%$ of the subjects could have lung cancer or a pre-invasive lesion diagnosed without additional risk.

\section{REFERENCES}

1 Woolner LB. Pathology of cancer detected cytologically. In: Woolner LB, ed. National Cancer Institute. Atlas of Early Lung Cancer. Tokyo, Igaku-Shoin, 1983; pp. 107-213.

2 Lam S, Profio AE. Fluorescence tumour detection. In: Hetzel MR, ed. Minimally Invasive Techniques in Thoracic Medicine and Surgery. London, Chapman \& Hall, 1995; pp. 179-191.

3 Lam S, Kennedy T, Unger M, et al. Localization of bronchial intraepithelial neoplastic lesions by fluorescence bronchoscopy. Chest 1998; 113: 696-702.

4 Lam S, MacAulay C, leRiche JC, Palcic B. Detection and localization of early lung cancer by fluorescence bronchoscopy. Cancer 2000; 89: Suppl. 11, 2468-2473.

5 Häußinger K, Becker H, Stanzel F, et al. Autofluorescence bronchoscopy with white light bronchoscopy compared with white light bronchoscopy alone for the detection of precancerous lesions: a European randomized controlled multicentre trial. Thorax 2005; 60: 496-503.

6 Saccomanno G, Archer VE, Auerbach O, Saunders RP, Brennan LM. Development of carcinoma of the lung as reflected in exfoliated cells. Cancer 1974; 33: 256-270.

7 The Japan Lung Cancer Society, Classification of Lung Cancer. 1st Edn. Tokyo, Kanehara \& Co, 2000.

8 Travis WD, Colby TV, Corrin B, et al. Histological typing of tumours of lung and pleura. In: Sobin LH, ed. World Health Organization International Classification of Tumours. 3rd Edn. Berlin, Springer-Verlag, 1999.

9 Jemal A, Tiwari RC, Murry T, et al. Cancer Statistics 2004. CA Cancer J Clin 2004; 54: 8-29.

10 Foo W, ed. Cancer Stat 2000. Hong Kong, Hong Kong Cancer Registry, Hospital Authority, 2003. 
11 Bota S, Auliac JB, Paris C, et al. Follow-up of bronchial precancerous lesions and carcinoma in situ using autofluorescence endoscopy. Am J Respir Crit Care Med 2001; 164: 1688-1693.

12 Sutedja TG, van Boxem AJ, Postmus PE. The curative potential of intraluminal bronchoscopic treatment for early-stage non-small-cell lung cancer. Clin Lung Cancer 2001; 2: 264-270.

13 Kennedy TC, Franklin WA, Prindiville SA, et al. High prevalence of occult endobronchial malignancy in high risk patients with moderate sputum atypia. Lung Cancer 2005; 49: 187-191.
14 van Rens MTM, Schramel FMNH, Elbers JRJ, Lamers JWJ. The clinical value of lung imaging fluorescence endoscopy for detecting synchronous lung cancer. Lung Cancer 2001; 32: 13-18.

15 Pierard P, Faber J, Hutsebaut J, et al. Synchronous lesions detected by autofluorescence bronchoscopy in patients with high grade pre-invasive lesions and occult invasive squamous cell carcinoma of the proximal airways. Lung Cancer 2004; 46: 341-347.

16 Berman JJ, Murray RJ, Lopez-Plaza IM. Widespread posttracheostomy atypia simulating squamous cell carcinoma. A case report. Acta Cytol 1991; 35: 713-716. 